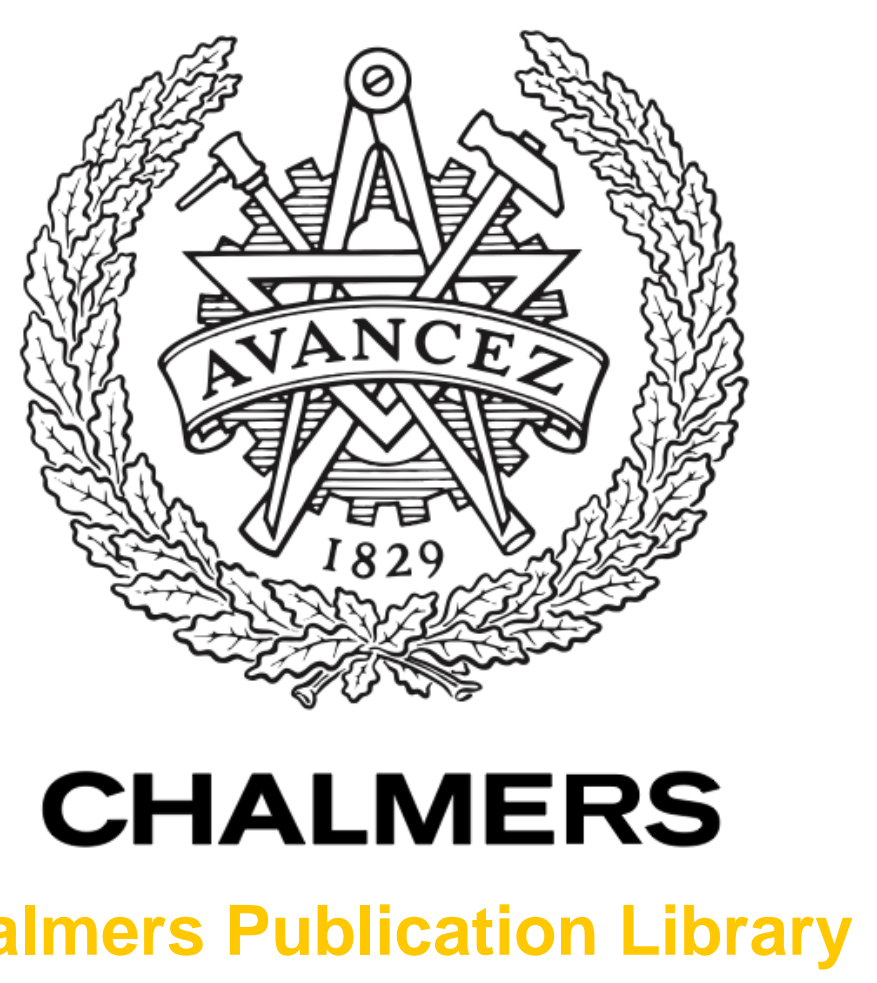

Chalmers Publication Library

\title{
Sensitivity Comparison of Time Domain Hybrid Modulation and Rate Adaptive Coding
}

This document has been downloaded from Chalmers Publication Library $(\mathrm{CPL})$. It is the author's version of a work that was accepted for publication in:

Optical fiber communication conference, 2016

Citation for the published paper:

Yan, L. ; Agrell, E. ; Wymeersch, H. (2016) "Sensitivity Comparison of Time Domain

Hybrid Modulation and Rate Adaptive Coding". Optical fiber communication conference, 2016 pp. W11-3.

Downloaded from: http://publications.lib.chalmers.se/publication/238426

Notice: Changes introduced as a result of publishing processes such as copy-editing and formatting may not be reflected in this document. For a definitive version of this work, please refer to the published source. Please note that access to the published version might require a subscription. 


\title{
Sensitivity Comparison of Time Domain Hybrid Modulation and Rate Adaptive Coding
}

\author{
Li Yan, Erik Agrell, and Henk Wymeersch \\ Department of Signals and Systems, Chalmers University of Technology, SE-41296 Gothenburg, Sweden \\ lyaa@chalmers.se
}

\begin{abstract}
We investigate the sensitivities using rate-adaptive coding (RAC), time domain hybrid modulation (TDHM), and their combination. We demonstrate that the sensitivity gains of TDHM and the combination are only marginal, if any, compared to RAC.
\end{abstract}

OCIS codes: (060.4510) Optical communications; (060.1660) Coherent communications.

\section{Introduction}

Driven by exponential growth of traffic demands, modern optical networks require more flexibility and capacity in transmitting high volume and heterogeneous data. Although higher-order polarization multiplexed (PM) quadrature amplitude modulation (QAM) has been proposed to increase spectral efficiency (SE), they only allow for coarse rate and transmission reach adaptation. Much finer SE and transmission reach tuning can be realized by either time domain hybrid modulation (TDHM) [1-5] or forward error correction (FEC) with rate adaptive coding (RAC) [6-9]. Since these two techniques serve essentially the same purpose to trade SE for sensitivity, one might ask which of them achieves the best trade-off.

In TDHM, two modulation frames are periodically alternated in time. By varying the duty cycles of the formats, any intermediate SE can be achieved [1]. Alternatively, a fine SE granularity can be realized by RAC and a fixed modulation format [6], where the trade-off between SE and transmission reach is achieved by adapting code redundancy. The combination of TDHM and RAC (TDHM+RAC) is proposed [10] to mix PM quadrature phase shift key (PM-QPSK) and PM-16QAM with different code rates to improve flexibility further.

Even though various experimental and numerical studies have been carried out to demonstrate improved flexibility and capacity of TDHM [2,3] and RAC [7-9], no sensitivity comparison between the two has been performed, which is the purpose of this paper.

We analyze the SNR thresholds of TDHM, RAC, and TDHM+RAC with both Reed-Solomon (RS) codes and capacity-achieving codes under hard-decision decoding, while optimizing the transmission parameters. Among the three schemes, RAC is almost always the most favorable one, whereas TDHM+RAC and TDHM achieve only marginal sensitivity gains in a few cases.

\section{Parameter Optimizations}

The TDHM scheme comprises two modulation formats: $F_{1}$ with $\mathrm{SE}_{1}$ and $F_{2}$ with $\mathrm{SE}_{2}$. By periodically transmitting $M_{1}$ symbols of $F_{1}$ and then switching to $M_{2}$ symbols of $F_{2}$ at the same symbol rate $R_{s}$, the overall $\mathrm{SE}$ is, assuming a fixed code rate $R_{c}$ [2,3], $\mathrm{SE}_{h}=R_{c}\left(\mathrm{FR} \cdot \mathrm{SE}_{1}+(1-\mathrm{FR}) \cdot \mathrm{SE}_{2}\right)$, where $\mathrm{FR}=M_{1} /\left(M_{1}+M_{2}\right)$ is the format ratio of $F_{1}$. Both modulation formats have their individual power $P_{i}$ and thus corresponding $\mathrm{SNR}_{\text {as }} \mathrm{SNR}_{i},(i=1,2)$. The individual pre-FEC BER is related to the SNR and modulation format as $\mathrm{BER}_{\text {pre }, i}=f\left(\mathrm{SNR}_{i} ; F_{i}\right),(i=1,2)$, where $f(\mathrm{SNR} ; F)$ calculates the uncoded BER for modulation $F$ in additive white Gaussian noise channel [11]. The post-FEC BER depends on specific coding scheme (RS or capacity-achieving codes in this paper) and code rate. After FEC decoding

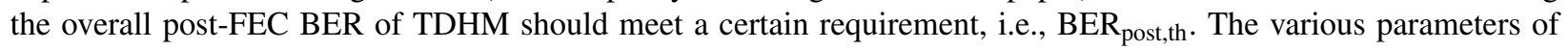

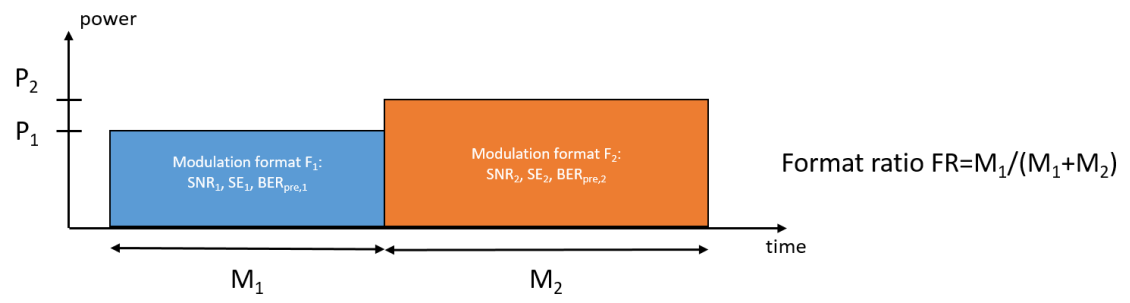

Fig. 1: Illustration of TDHM and its parameters in time domain. 
TDHM are summarized in Figure 1. Once the pair of modulation formats, the targeted overall $\mathrm{SE}_{h}$, and the code rate $R_{C}$ are given, the format ratio is fixed. By optimizing the transmit powers of both modulations, the SNR threshold can be obtained [4].

In RAC, given a coding scheme, a fixed modulation format $F_{1}$ with spectral efficiency $\mathrm{SE}_{1}$, and an overall target $\mathrm{SE}$, the code rate is fixed as $R_{c}=\mathrm{SE} / \mathrm{SE}_{1}$. The pre-FEC BER threshold is $\mathrm{BER}_{\text {pre,th }}=g\left(\mathrm{BER}_{\mathrm{post}, \mathrm{t}}, R_{c}\right)$, where $g\left(\mathrm{BER}_{\text {post,th }}, R_{c}\right)$ is a function of the error correction capability of the chosen code rate and the post-FEC BER threshold. $\mathrm{BER}_{\text {pre,th }}$ is then translated to an SNR threshold through $\mathrm{SNR}=f^{-1}\left(\mathrm{BER}_{\text {pre,th }} ; F_{1}\right)$. By selecting modulation with the lowest SNR threshold for each value of SE, we can obtain the most sensitive RAC scheme [7].

For TDHM+RAC, we can vary the format ratio, the two transmit powers, and the code rate. Unlike the flex-FEC scheme [10], which applies different FEC codes to the two formats, we use a single code rate in all cases. This is for complexity reasons and because the two modulations are switched quite frequently in TDHM [2-4]. The number of bits in one modulation frame is typically much smaller than the 1000 or 10000 bits in a FEC codeword.

The set of parameters that gives the SNR threshold is found through the following optimization problem. The inputs are $\mathrm{SE}_{h}, \mathrm{SE}_{1}, \mathrm{SE}_{2}, \mathrm{BER}_{\text {post,th }}$ and the functions $f\left(\cdot ; F_{1}\right), f\left(\cdot ; F_{2}\right)$, and $g(\cdot)$, while the outputs are the optimal $\mathrm{FR}, R_{c}, \mathrm{SNR}_{1}$ and $\mathrm{SNR}_{2}$ :

$$
\begin{array}{ll}
\underset{\mathrm{FR},,_{c},}{\operatorname{minimize}} \mathrm{SNR}_{1} \mathrm{SNR}_{2} & \\
\text { subject to } & \mathrm{SNR}_{h}=\mathrm{FR} \cdot \mathrm{SNR}_{1}+(1-\mathrm{FR}) \cdot \mathrm{SNR}_{2} \\
& \mathrm{SE}_{1} \cdot \mathrm{FR} \cdot R_{c}+\mathrm{SE}_{2} \cdot(1-\mathrm{FR}) \cdot R_{c}=\mathrm{SE} \\
& \mathrm{BER}_{\mathrm{pre}, i}=f\left(\mathrm{SNR}_{i} ; F_{i}\right), \quad i=1,2 \\
& \mathrm{BER}_{h}=\frac{\mathrm{SE}_{1} \cdot \mathrm{FR}_{i} \cdot \mathrm{BER}_{\mathrm{pre}, 1}+\mathrm{SE}_{2} \cdot(1-\mathrm{FR}) \cdot \mathrm{BER}_{\mathrm{pre}, 2}}{\mathrm{SE}_{1} \cdot \mathrm{FR}+\mathrm{SE}_{2} \cdot(1-\mathrm{FR})} \\
& \mathrm{BER}_{h} \leq \mathrm{BER}_{\mathrm{pre}}, \mathrm{th} \\
& \mathrm{BER}_{\mathrm{pre}, \text { th }}=g\left(\mathrm{BER}_{\mathrm{post}, \mathrm{th}}, R_{c}\right) .
\end{array}
$$

(1a) calculates the overall SNR. (1b) imposes that the target SE requirement should be satisfied. (1c) calculates preFEC BERs from SNRs. In (1d) and (1e) the overall pre-FEC BER is calculated and required to be lower than the

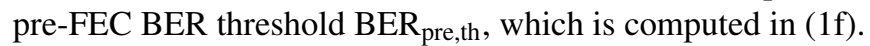

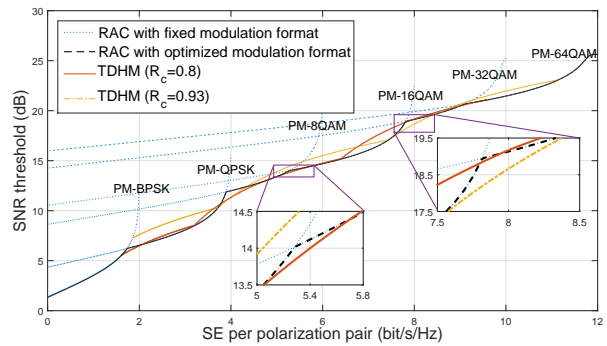

(a)

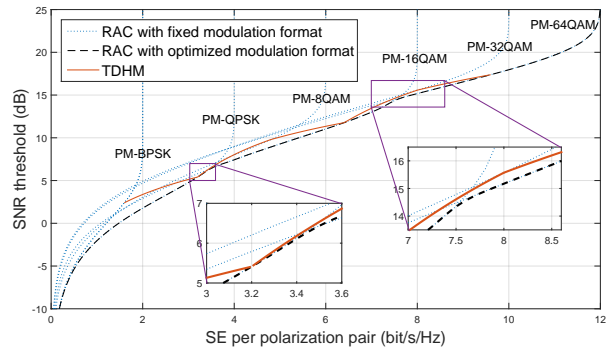

(b)

Fig. 2: Required SNR for RAC and TDHM with (a) RS codes and (b) ideal capacity-achieving codes. The maximal sensitivity gain of TDHM is $0.8 \mathrm{~dB}$, as highlighted by the ellipse in (a). TDHM is mainly RAC occasionally in the RS code case, whereas RAC is always better than TDHM if capacity-achieving code is used.

\section{Sensitivity Comparison}

The parameter optimization for all the schemes is implemented numerically. The available modulations are PM-BPSK, PM-QPSK, PM-8QAM, PM-16QAM, PM-32QAM, and PM-64QAM. For TDHM, modulations with adjacent orders are mixed for intermediate SEs. The post-FEC threshold is $10^{-9}$.

First, the sensitivities of RAC and TDHM under RS codes and capacity-achieving codes are compared in Figure 2. In RAC, the SNR thresholds for every fixed modulation format are first calculated (blue dotted curves in Figure 2). Then the modulation with the highest sensitivity is selected for each value of SE (black dashed curve in Figure 2).

With the RS codes, two code rates are selected for TDHM, i.e., $R_{c}=0.8$ and $R_{c}=0.93$. As shown in Figure 2a, both code rates of TDHM have higher SNR thresholds than RAC at most of the SEs, while it outperforms RAC only when modulation format is switched in RAC. As highlighted by the ellipse in Figure 2a, the highest sensitivity 
gains of TDHM with both code rates are $0.8 \mathrm{~dB}$. The insets in Figure 2a illustrate that TDHM with $R_{c}=0.8$ is more sensitive than RAC at low SE region whereas high code rate TDHM $\left(R_{c}=0.93\right)$ is preferred at high SE region. With the capacity-achieving codes, however, Figure $2 \mathrm{~b}$ shows that TDHM always requires higher SNR threshold than RAC. The insets of Figure $2 b$ indicates that even at the modulation switching points, the SNR thresholds of RAC are still lower than TDHM. Envisioning advanced FEC with high performance, RAC is more favorable than TDHM.

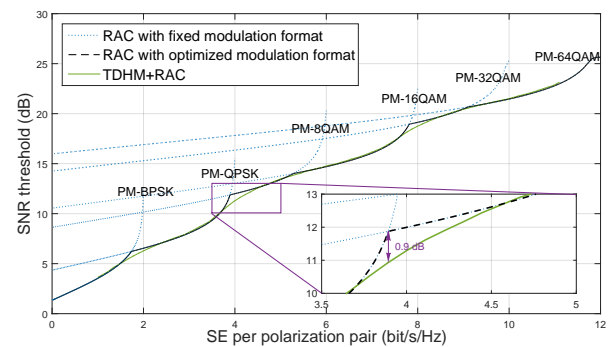

Fig. 4: The SNR threshold for RAC and TDHM+RAC with RS codes. TDHM+RAC is preferred around the modulation switching points of RAC.

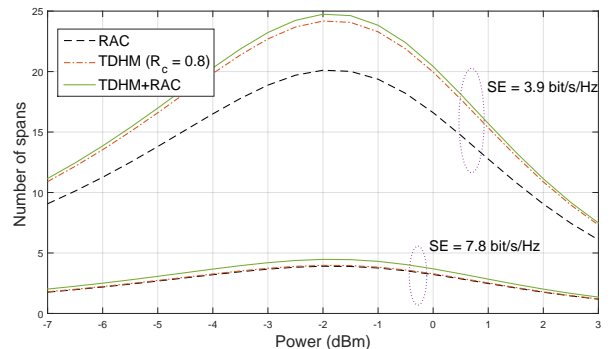

Fig. 5: Transmission reach of all schemes with RS codes for $\mathrm{SE}=$ 3.9 and $7.8 \mathrm{bit} / \mathrm{s} / \mathrm{Hz}$, respectively. The reach is extended by 4 spans with hybrid modulation at low SE, and less than 1 span at high SE.

TDHM+RAC has similar performance as TDHM. The results are shown in Figure 4. In the RS case, the SNR threshold of the TDHM+RAC is slightly lower than the RAC scheme around the modulation switching points, and has identical performance elsewhere. The maximum sensitivity gain of TDHM+RAC compared with RAC is $0.9 \mathrm{~dB}$. In the capacity-achieving code case, however, TDHM+RAC performs identically as RAC because the format ratio of the lower order modulation is always 0 .

The sensitivities under RS codes are then evaluated by transmission reach, which is calculated based on Gaussian noise model [12]. We assume dispersion uncompensated fiber link with $100 \mathrm{~km}$ span length and EDFA with $5.5 \mathrm{~dB}$ noise figure. The fiber attenuation is $\alpha=0.22 \mathrm{~dB} / \mathrm{km}$, dispersion is $D=16.7 \mathrm{ps} / \mathrm{nm} / \mathrm{km}$, and nonlinear coefficient $\gamma=$ $1.3(\mathrm{~W} \cdot \mathrm{km})^{-1} .9$ channels with the same power are co-propagated. As shown in Figure 5, for $\mathrm{SE}=3.9 \mathrm{bit} / \mathrm{s} / \mathrm{Hz}$, both TDHM and TDHM+RAC extend the transmission reach by 4 spans compared with RAC. But they perform similarly when $\mathrm{SE}=7.8 \mathrm{bit} / \mathrm{s} / \mathrm{Hz}$. With capacity-achieving codes, however, all three schemes have very similar reaches.

The reason why TDHM and TDHM+RAC schemes can outperform RAC with the RS codes is that the code rate changes significantly when modulation format is switched in the RAC scheme, which results in large variation of error correction capability as well. Consequently, the SNR threshold curve first increases rapidly and then slows down immediately around the turning points. In contrast, by adjusting frame ratio or code rate in TDHM and TDHM+RAC, the abruptly changing SNR threshold curve is smoothed. Hence the sensitivity of TDHM is superior to RAC around the turning points. However, since the degeneration of error correction capability at high code rate for capacity-achieving codes is not as significant as the RS codes, sharp turning points disappear in Figure 2b. Hence the advantages of TDHM and TDHM+RAC vanish in this situation.

\section{Conclusion}

TDHM is not an alternative modulation technique in systems that employ variable-rate FEC coding. The same gains in terms of flexible SE that TDHM offers can be obtained by varying the code rate, which in general yields better sensitivity than TDHM.

\section{References}

1. X. Zhou, et. al., "Rate-adaptable optics for next generation long-haul transport networks." IEEE Commun. Mag., 2013.

2. F. Buchali, et. al. "Performance and advantages of $100 \mathrm{~Gb} / \mathrm{s}$ QPSK/8QAM hybrid modulation formats." OFC, 2015.

3. F. Buchali, et. al., "Hybrid modulation formats outperforming 16QAM and 8QAM in transmission distance and filtering with cascaded WSS." OFC, 2015.

4. V. Curri, et. al., "Time-division hybrid modulation formats: Tx operation strategies and countermeasures to nonlinear propagation." OFC, 2014.

5. A. Ahmad, et. al., "Impact of fiber types and raman pumping in reconfigurable DWDM transparent optical networks." ECOC, 2015.

6. D. Mello, et. al., "Optical networking with variable-code-rate transceivers.” J. Lightw. Technol., 2014.

7. J. Zhao, et. al., "Code rate optimization in elastic optical networks." ECOC, 2015.

8. N. Sambo, et. al., "Routing, code, and spectrum assignment (RCSA) in elastic optical networks." OFC, 2015.

9. A. Alvarado, et. al., "On optimal modulation and FEC overhead for future optical networks." OFC, 2015.

10. A. Arduino, et. al., "Flexible FEC optimization for time-domain hybrid modulation formats." SPPCom, 2015.

11. Cho, K., et. al., "On the general BER expression of one- and two-dimensional amplitude modulations”, IEEE Trans. Commun., 2002.

12. P. Johannisson, and E. Agrell. "Modeling of nonlinear signal distortion in fiber-optic networks." JLT, 2014. 